\title{
Elevated X-linked inhibitor of apoptosis protein (XIAP) expression uncovers detrimental prognosis in subgroups of neoadjuvant treated and T-cell rich esophageal adenocarcinoma
}

\author{
Lars M. Schiffmann ${ }^{1,2,3^{*}+}$ (1), Heike Göbel ${ }^{3,4 \dagger}$, Heike Löser ${ }^{3,4}$, Fabian Schorn ${ }^{1}$, Jan Paul Werthenbach ${ }^{1}$, Hans F. Fuchs ${ }^{2,3}$, \\ Patrick S. Plum ${ }^{2,3}$, Marc Bludau ${ }^{2,3}$, Thomas Zander ${ }^{3,5}$, Wolfgang Schröder ${ }^{2,3}$, Christiane J. Bruns ${ }^{2,3}$, Hamid Kashkar 1,3, \\ Alexander Quaas ${ }^{3,4+}$ and Florian Gebauer ${ }^{2,3+}$
}

\begin{abstract}
Background: Molecular markers predicting survival in esophageal adenocarcinoma (EAC) are rare. Specifically, in favorable oncologic situations, e.g. nodal negativity or major neoadjuvant therapy response, there is a lack of additional risk factors that serve to predict patients' outcome more precisely. This study evaluated X-linked inhibitor of apoptosis protein (XIAP) as a potential marker improving outcome prediction.
\end{abstract}

Methods: Tissue microarrays from 362 patients that were diagnosed with resectable EAC were included in the study. XIAP was stained by immunohistochemistry and correlated to clinical outcome, molecular markers and markers of the cellular tumor microenvironment.

Results: XIAP did not impact on overall survival (OS) in the whole study collective. Subgroup analyses stratifying for common genetic markers (TP53, ERBB2, ARID1A/SWI/SNF) did not disclose any impact of XIAP expression on survival. Detailed subgroup analyses of [1] nodal negative patients, [2] highly T-cell infiltrated tumors and [3] therapy responders to neoadjuvant treatment revealed a significant inverse role of high XIAP expression in these specific oncologic situations; elevated XIAP expression detrimentally affected patients' outcome in these subgroups. [1]: OS XIAP low: 202 months (m) vs. XIAP high: 38 m; [2]: OS 116 m vs. 28.2 m; [3]: OS 31 m vs. 4 m).

Conclusions: Our data suggest XIAP expression in EAC as a worthy tool to improve outcome prediction in specific oncologic settings that might directly impact on clinical diagnosis and treatment of EAC in the future.

Keywords: XIAP, EAC, Biomarker of response, Outcome prediction

* Correspondence: lars.schiffmann@uk-koeln.de

${ }^{\dagger}$ Lars M Schiffmann and Heike Göbel share first authorship

${ }^{\dagger}$ Alexander Quaas and Florian Gebauer share senior authorship

${ }^{1}$ Cologne Excellence Cluster on Cellular Stress Responses in Aging-Associated

Diseases (CECAD), Center for Molecular Medicine Cologne (CMMC) and Institute for Medical Microbiology, Immunology and Hygiene, University of Cologne, CECAD Research Center, Joseph-Stelzmann-Str. 26, 50931 Cologne, Germany

${ }^{2}$ Department of General, Visceral and Cancer Surgery, University of Cologne, Kerpener Str. 62, 50937 Cologne, Germany

Full list of author information is available at the end of the article

\section{Background}

Esophageal adenocarcinoma (EAC) shows an increasing incidence over the last decades in the western world [1]. Multi-disciplinary treatment strategies including intense neoadjuvant treatment regimens and radical oncologic surgery continually contribute to improved survival rates. Though overall prognosis is limited and EAC ranks on 6th place for cancer associated death [2]. Most patients are diagnosed with advanced tumor stages including presence of lymph node metastasis and locally advanced tumor stages. Since the tumor infiltration depth $(\mathrm{pT})$ and presence of

(C) The Author(s). 2019 Open Access This article is distributed under the terms of the Creative Commons Attribution 4.0 International License (http://creativecommons.org/licenses/by/4.0/), which permits unrestricted use, distribution, and 
lymph node metastasis are the main pathological factors predicting long-term survival in EAC, within particular pathological subgroups, the overall-survival differs significantly without prior knowledge of the individual patients' prognosis. For this reason, there is a mandatory need for the identification of biomarkers allowing the stratification of patients with similar $\mathrm{pT}$ and $\mathrm{pN}$ stages into high- and low-risk patients.

The X-linked inhibitor of apoptosis protein (XIAP) has been frequently shown to be upregulated in different cancer entities [3-6]. Besides its anti-apoptotic function [7] XIAP was additionally shown to promote cellular inflammatory signaling and trigger cytokine secretion [8-10].

The objective of this study is to assess the significance of XIAP expression as a predictor of overall survival in EAC. We analyzed XIAP in the largest collective of EAC so far. Our data show for the first time that in different generally favorable clinical situations (tumor response to neoadjuvant therapy, in nodal negativity and in highly T-cell infiltrated tumors) XIAP expression can be used to identify patients that have a poor prognosis which is not predictable with current state of the art staging methods.

\section{Methods}

\section{Patients and tumor samples}

Formalin-fixed and paraffin embedded material of 362 patients with esophageal adenocarcinomas that underwent primary surgical resection or resection after neoadjuvant therapy between 1999 and 2014 at the Department of General, Visceral and Cancer Surgery, University of Cologne, Germany were analyzed. Standard surgical procedures were either transthoracic esophagectomy with lymphadenectomy of the mediastinal and abdominal compartment (2-field LAD), transhiatal esophagectomy with lymphadenectomy of the lower mediastinum or transhiatal extended gastrectomy with D2-lymphadenectomy.

Patients with locally advanced esophageal cancer (cT3) or evidence for locoregional lymph node metastasis in clinical staging received preoperative chemoradiation or chemotherapy according to established protocols within national guidelines [11-14].

Construction of the tissue-micro arrays (TMA) was performed as previously described $[15,16]$. In brief, tissue cylinders with a diameter of $1.2 \mathrm{~mm}$ each were punched from selected tumor tissue blocks using a self-constructed semi-automated precision instrument and embedded in empty recipient paraffin blocks. $4 \mu \mathrm{m}$ sections of the resulting TMA blocks were transferred to an adhesive coated slide system (Instrumedics Inc., Hackensack, NJ) for immunohistochemistry (IHC).

All procedures performed in studies involving human participants were in accordance with the ethical standards of the institutional research committee and with the 1964
Helsinki declaration and its later amendments or comparable ethical standards.

\section{Immunohistochemistry (IHC)}

IHC was performed on TMA slides. Tumor cell XIAP was detected using a polyclonal rabbit anti-XIAP antibody (ab21278: dilution 1:1000) on Leica BOND-MAX stainer (Leica Biosystems, Germany) according to the manufacturers' protocol.

We correlated the XIAP results with previously collected and described data like T-cell inflammation of the tumor microenvironment and different molecular tumor cell alterations like TP53, ARIDa1 loss and ERBB2- amplification $[17,18]$.

\section{Analysis}

The evaluation of immunohistochemical expression scores was peformed manually by high-level trained pathologists (HG, HL) independently and in a blinded fashion to any clinical details. The following scores were used for the analysis:

XIAP: no staining was considered as negative (0), a weak staining intensity in less than $70 \%$ or a moderate staining intensity in less than $30 \%$ of tumor cells was considered as weak [1], a moderate staining intensity in less than $70 \%$ or a weak staining intensity in more than $70 \%$ or a high tumor intensity in less than $30 \%$ of tumor cells as moderate [2] and a high staining intensity in more than $30 \%$ of the tumor cells or moderate intensity in more than $70 \%$ was considered as high [3].

\section{Statistical analysis}

Clinical data were collected prospectively according to a standardized protocol. SPSS Statistics for Mac (Version 24, IBM) was used for statistical analysis. Interdependence between stainings and clinical data were calculated using Fisher's exact tests. Survival curves were plotted using the Kaplan-Meier method and analyzed using the log-rank test. All tests were two-sided. $P$ values $<0.05$ were considered statistically significant.

\section{Results}

TMA spots from 362 resected EACs (UICC stages I-III) were stained for XIAP. 311 tumor-containing spots (85.9\%) were eligible to be scored for XIAP expression by a pathologist blinded to any clinical details. Reasons for non-informative cases (51 spots; 14.1\%) included lack of tissue samples or absence of unequivocal cancer tissue in the TMA spot. XIAP was determined negative in 28 cases (7.7\%). Score 1 was detected in 132 patients (36.5\%), score 2 in 107 (29.6\%) and score 3 in 44 patients (12.2\%). For further analyses, patients were stratified for score $0-2$ which was summarized as XIAP low $(n=267$, $85.9 \%$ ) and score 3 which was considered to be XIAP 
high $(n=44,14.1 \%)$. Public domain data analyses revealed high mRNA levels for XIAP in $9.2 \%$ of the cases for EAC (data not shown) which validates our findings $[19,20]$. Representative images of XIAP low and XIAP high tumors are depicted in Additional file 4: Figure S4. Table 1 depicts clinical characteristics of these 311 XIAP low or high patients included in the study.

In XIAP low versus XIAP high expressing tumors the overall-survival (OS) did not significantly differ in the complete study collective. Median OS was 32.5 months (95\% CI 24.9-40.0 months) in the XIAP low compared

Table 1 Basic clinical and demographic characteristics of studied patients.

\begin{tabular}{lllllll}
\hline total & & XIAP low & & XIAP high & p \\
\hline$n$ & $\%$ & $n$ & $\%$ & $n$ & $\%$ &
\end{tabular}

\begin{tabular}{|c|c|c|c|c|c|c|c|}
\hline number of patients & 311 & 100 & 267 & 85.9 & 44 & 14.1 & \\
\hline \multicolumn{8}{|l|}{ sex } \\
\hline female & 32 & 10.3 & 29 & 90.6 & 3 & 9.4 & 0.593 \\
\hline male & 279 & 89.7 & 238 & 85.3 & 41 & 14.7 & \\
\hline age at diagnosis & & & 62.1 & & 66.3 & & 0.032 \\
\hline \multicolumn{8}{|l|}{ initial T stage $\left({ }^{(a}\right)$} \\
\hline pT1 & 25 & 8.1 & 21 & 84 & 4 & 16 & 0.151 \\
\hline pT2 & 29 & 9.4 & 29 & 100 & 0 & 0 & \\
\hline pT3 & 250 & 80.6 & 211 & 84.4 & 39 & 15.6 & \\
\hline pT4 & 6 & 1.9 & 5 & 83.3 & 1 & 16.7 & \\
\hline
\end{tabular}

initial $N$ stage $\left({ }^{a}\right)$

$\begin{array}{clllllll}\text { pN0 } & 126 & 40.6 & 104 & 82.5 & 22 & 17.5 & 0.260 \\ \text { pN1 } & 113 & 36.5 & 101 & 89.4 & 12 & 10.6 & \\ \text { pN2 } & 36 & 11.6 & 29 & 80.6 & 7 & 19.4 & \\ \text { pN3 } & 35 & 11.3 & 32 & 91.4 & 3 & 8.6 & \\ \text { Grading } & & & & & & & \\ \text { G1 } & 3 & 1.4 & 3 & 100 & 0 & 0 & 0.539 \\ \text { G2 } & 136 & 62.4 & 112 & 82.4 & 24 & 17.6 & \\ \text { G3 } & 78 & 35.8 & 69 & 88.5 & 9 & 11.5 & \\ \text { G4 } & 1 & 0.5 & 1 & 100 & 0 & 0 & \end{array}$

R status $\left({ }^{\mathrm{C}}\right)$

$\begin{array}{llllllll}\text { R0 } & 206 & 66.2 & 175 & 85 & 31 & 15 & 0.424 \\ \text { R1 } & 16 & 5.1 & 14 & 87.5 & 2 & 12.5 & \\ \text { R2 } & 2 & 0.6 & 1 & 50 & 1 & 50 & \end{array}$

neoadjuvant therapy $\left(^{(b)}\right.$

$\begin{array}{llllllll}\text { yes } & 185 & 60.3 & 165 & 89.2 & 20 & 10.8 & 0.064 \\ \text { no } & 122 & 39.7 & 99 & 81.1 & 23 & 18.9 & \end{array}$

type of neoadj. Therapy (')

\begin{tabular}{llllllll} 
Retx & 107 & 93 & 86 & 80.4 & 21 & 19.6 & 0.620 \\
Ctx & 8 & 7 & 7 & 87.5 & 1 & 12.5 & \\
\hline
\end{tabular}

$\mathrm{a}, \mathrm{bc}$, indicate missing clinical information for the respective category $\left({ }^{\mathrm{a}}: n=1\right.$ missing, ${ }^{b}: n=4$ missing, ${ }^{c} n=87$ missing, ${ }^{\prime}=74$ missing) to 38.0 months (95\% CI 23.2-52.7 months, $p=0.775$ ) in the XIAP high group (Fig. 1).

As XIAP is thought to be involved in therapy resistance [5] due to decreased cell death in XIAP overexpressing tumors we investigated whether XIAP levels can be correlated to treatment response (to combined radio- chemotherapy or chemotherapy alone in a neoadjuvant fashion) measured by a histopathological regression score. Regression scores were available from 133 patients of the eligible patients. Histopathological regression score ranged from 1 to 3 ; 1 was considered as non-responder, 2 and 3 as responders (modified from [21]). Histopathological non-responders did not have a higher XIAP score per se indicating that XIAP in EAC does not result in increased resistance to neoadjuvant therapy (Table 2).

When we plotted survival stratified for therapy response we saw that XIAP expression in therapy responders is capable of identifying patients that have a worse overall prognosis though they responded to therapy. Median OS in XIAP low, therapy responsive patients was 26.3 months (95\% CI $0.0-18.5$ months) compared to 3.3 months (95\% CI 0.0-26.2 months, $p=0.036$, Fig. 1b).

This led us to the hypothesis that XIAP might be more important in general in neoadjuvant treated patients than in chemo and/or radiation naïve patients. According to national and international guidelines neoadjuvant therapy is conducted in locally advanced stages (cT3, cT4). We therefore performed subgroup analysis for T3 and T4 patients. In general, also in this group, XIAP expression did not affect overall survival (XIAP low: median OS 30.5 months (95\% CI $24.3-36.75$ months) vs. XIAP high: 33.8 months (95\% CI 17.1-50.5 months; $p=$ $0.568)$ ) but was able to predict poor outcome in therapy responders (XIAP low: median OS 30.5 months $(95 \% \mathrm{CI}$ 22.4-38.6 months) vs. XIAP high: 3.3 months (95\% CI 0.0-28.5 months; $p=0.026)$ ), (Fig. 1c, d).

On this basis we hypothesized that XIAP might be useful to define high risk situations in certain oncologic situations. We therefore analysed other specific subgroups that in general are believed to be favorable. First, we looked for nodal status. Patients that were found to be nodal negative had a significantly shortened survival when their intratumoral XIAP expression was high. OS in nodal negative, XIAP low patients was 202.2 months (95\% CI 64.8-339.6 months, $p=0.546)$ while OS dropped drastically to 38.0 month in nodal negative, XIAP high patients (95\% CI $0.00-94.8$ months, $p=$ 0.022), (Fig. 2).

Based on its capability to interfere with cellular death while promoting inflammatory signaling we hypothesized that XIAP might be particularly important in an immunoreactive tumor environment which is characterized by tumor infiltrating T-cells which execute a tumoricidal immune response under certain conditions. We stratified the 


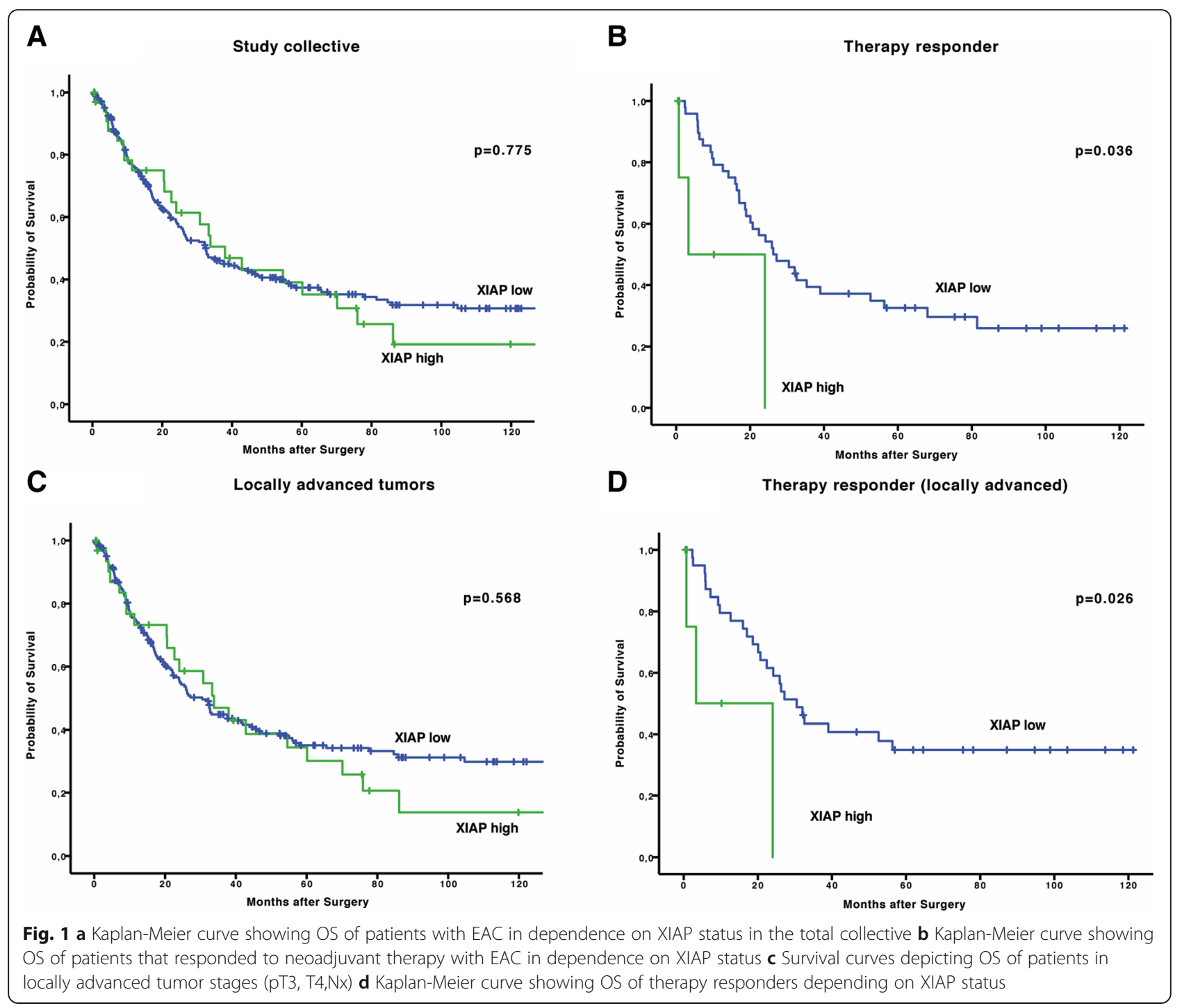

patient collective for CD3 low and CD3 high tumors as CD3 positive T-cell infiltration is considered to be another setting of superior overall outcome [22].

We found that in highly T-cell infiltrated tumors (CD3 high) high XIAP expression is detrimental for overall survival. Patients in this subgroup with low XIAP scores had a mean OS of 116.1 months (95\% CI 89.6-142.6 months) whereas in patients with XIAP high tumors mean OS was 28.2 months (95\% CI 9.7-46.6 months, $p=0.010$ ), (Fig. 3a). This correlation was not apparent in CD3 low tumors

Table 2 Cross-table showing distribution of therapy responders stratified for XIAP low vs. XIAP high expression

\begin{tabular}{llllll}
\hline & & low & high & Total & $\mathrm{p}$ \\
\hline Response & no & 65 & 11 & 76 & \\
& yes & 51 & 6 & 57 & 0.604 \\
& Total & 116 & 17 & 133 & \\
\hline
\end{tabular}

(median OS 63.3 months (48.9-77.7) vs. 54.0 months (34.1-73.9), $p=0.502)$ (Fig. 3b).

To further verify our findings, we conducted multivariate analyses. In a cox-regression model of the whole study cohort, nodal status and grading were independent prognostic factors, XIAP was not associated with survival as expected. Within the CD3 high subgroup grading and XIAP (instead of nodal status) were independent prognostic factors for overall survival proving the significant role of XIAP in this subgroup (Table 3).

Our findings suggest that the tumor microenvironment is crucially involved in the effect of tumor cell XIAP on patient's outcome. To strengthen this point we stratified for tumor cell genetic markers such as TP53, ERBB2 and ARID1A/SWI/SNF which would disregard corresponding tumor cell endogenous signaling pathways as the main driver of these effects. In fact, within any of these subgroups XIAP did not show an impact on overall survival 


\section{Nodal negative patients}

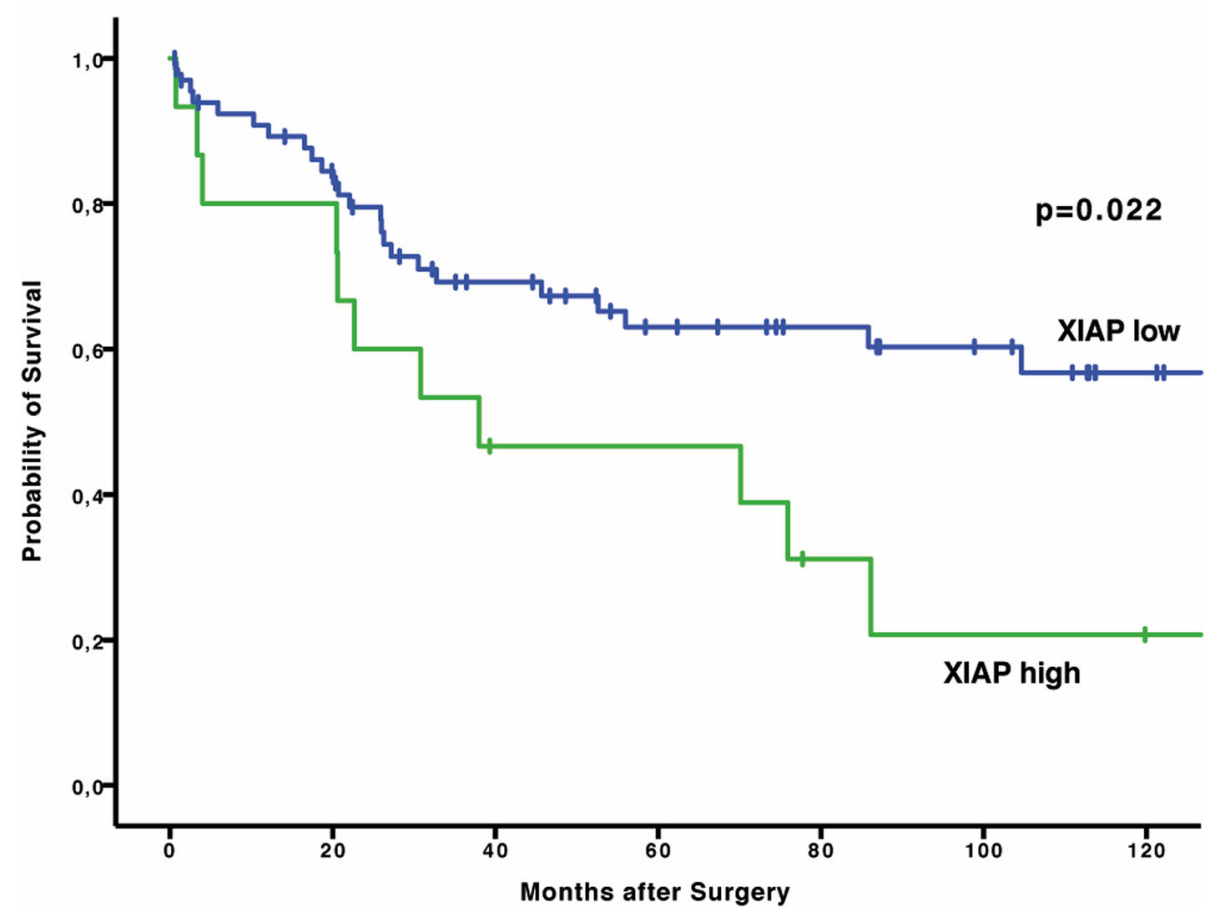

Fig. 2 Kaplan-Meier curve showing OS of patients with nodal negative EAC stratified for XIAP low vs. high

(Additional file 1: Figure S1, Additional file 2: Figure S2, Additional file 3: Figure S3).

\section{Discussion}

In the present study, we analyzed the protein expression of XIAP in a large and well characterized collective of esophageal adenocarcinomas. So, it was possible to perform subgroup analyses that could show the influence of XIAP on overall survival in specific patient groups. We could identify high levels of XIAP expression in malignant cells as a predictor of an immense negative prognosis in patients with nodal negative EAC who are at large considered to have a favorable outcome. We could furthermore show that elevated XIAP
A

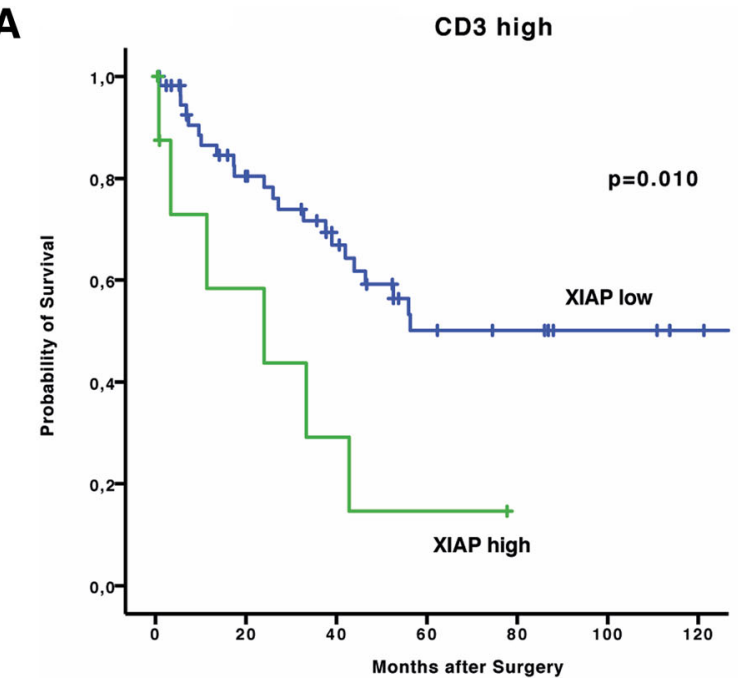

B

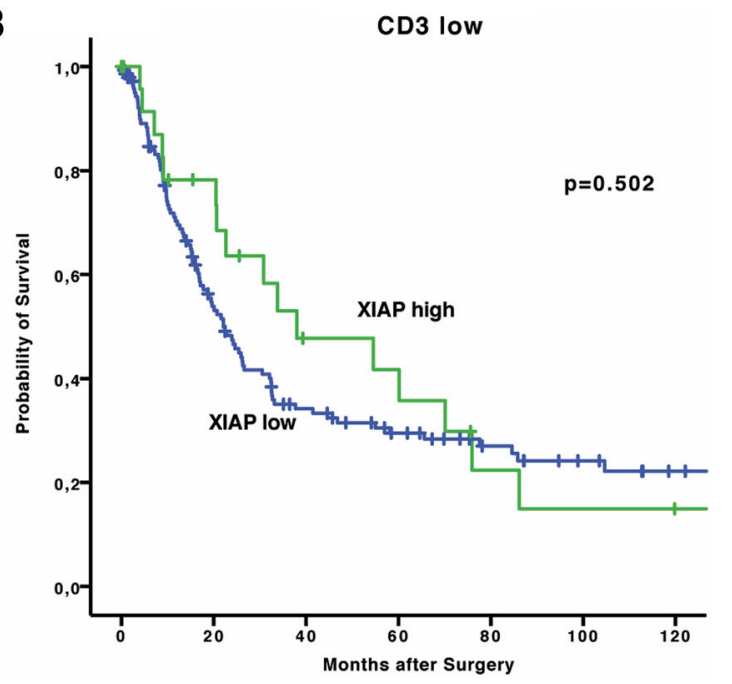

Fig. 3 Survival curves depicting OS of patients with high (a) or low (b) T-cell infiltrated tumors (XIAP low vs. high tumors) 
Table 3 Multivariate analyses of the whole study cohort and the CD3 high subgroup

\begin{tabular}{llllll}
\hline & & & \multicolumn{3}{c}{$95 \%$ confidence interval } \\
\cline { 5 - 6 } & & HR & lower & upper & $p$-value \\
\hline study cohort & sex & 1.673 & 0.773 & 3.623 & 0.192 \\
& age & 1.174 & 0.797 & 1.728 & 0.416 \\
& nodal status & 2.900 & 1.886 & 4.458 & $<0.001$ \\
& grading & 1.843 & 1.279 & 2.657 & 0.001 \\
& XIAP & 1.223 & 0.686 & 2.181 & 0.494 \\
CD3 high subgroup & sex & 0.598 & 0.102 & 3.501 & 0.568 \\
& age & 0.595 & 0.205 & 1.731 & 0.341 \\
& nodal status & 1.678 & 0.663 & 4.248 & 0.275 \\
& grading & 3.792 & 1.193 & 12.056 & 0.024 \\
& XIAP & 8.367 & 1.589 & 44.072 & 0.012 \\
\hline
\end{tabular}

expression compromises overall survival in patients with highly $\mathrm{T}$-cell infiltrated tumors that normally have a promising prognosis [22] and that patients that respond to chemotherapy and have high XIAP protein levels are at high risk.

Other studies previously reported a survival benefit for patients with lower expression of XIAP in comparison with patients expressing high levels of XIAP in esophageal squamous cell carcinoma (ESCC) but were not able to reveal a difference in OS in esophageal adenocarcinoma [23]. In contrast to the current study, only a relatively small group of patients was examined in which, due to the limited number of patients, sufficient subgroup analyses may not lead to significant results.

There are several possible scenarios that could explain our observation on a molecular level. Tumor cells with pathologically elevated XIAP levels are known to be more resistant to radio- or chemotherapy by reducing the capability to undergo therapy induced cell death [5]; it was already shown that reducing XIAP levels can re-sensitize cells to therapy induced apoptosis [24]. Since in our study collective, high XIAP expression was not correlated to a decrease in response to neoadjuvant therapy the observed effect cannot be explained by XIAP promoted therapy resistance only. In fact, we could observe that XIAP was therapy relevant in patients that are nodal negative, highly infiltrated by CD3 positive T-cells and had responded to therapy. It is therefore reasonable to think that the underlying mechanism is more complex and involves the tumor microenvironment rather than it is simply resistance to cell death that determines patients' outcome, which is strengthened by the fact that expression/mutational status of conventional genetic markers did not influence XIAPs role in predicting overall survival.

Hypothetically cancer cell XIAP is involved in an immunomodulatory process that mitigates T-cell antitumor activity as XIAP is described to be involved in $\mathrm{NFKB}$ signaling [25, 26]. XIAP overexpression could result in constitutively activated NFKB leading to increased cytokine secretion that e.g. can recruit immune cells suppressing T-cell response or directly impede T-cell cytotoxicity [27]. Whether lymphangiogenesis as one prerequisite of nodal metastasis is involved in XIAP-related mechanisms and how immune cell infiltration besides T-cells is altered in XIAP low vs. XIAP high tumors is among the questions to be answered in the future. Anyhow, recently developed novel drugs that selectively target the pro-inflammatory properties of XIAP may provide an innovative therapeutic strategy in patients with EAC $[26,28]$.

\section{Conclusions}

According to our data, XIAP could serve to identify high-risk patients within clinical low-risk groups according to the TNM staging system used so far. Those patients could be offered more aggressive therapy options (e.g. in XIAP high in nodal negative patients) or undergo extended surveillance after therapy. Since the current study is of retrospective character, future work should focus on prospective studies examining the impact of XIAP expression in specific patient subgroups. Additionally, pharmacological XIAP antagonization should be further evaluated in preclinical studies to evaluate its potential as targeted cancer therapy.

\section{Additional files}

Additional file 1: Figure S1. Kaplan-Meier curve showing OS of patients with either wildtype TP53 (A) or mutated TP53 (B) stratified for XIAP low vs. high. (A) XIAP low: median OS 32.7 months (95\% CI 17.9-47.6 months) vs. XIAP high: 33.3 months (95\% CI 16.5-50.1 months). (B) XIAP low: median OS 30.5 months ( $95 \%$ Cl 22.5-38.5 months) vs. XIAP high: 42.8 months (95\% Cl 23.3-62.3 months). (TIF 6349 kb)

Additional file 2: Figure S2. Kaplan-Meier curve showing OS of patients with either non-amplified erbb2 (A) or amplified erbb2 (B) stratified for XIAP low vs. high. (A) XIAP low: median OS 25.4 months (95\% CI 19.131.7 months) vs. XIAP high: 30.8 months ( $95 \%$ Cl 14.0-47.6 months). (B) XIAP low: median OS 55.0 months ( $95 \%$ Cl 41.6-68.4 months) vs. XIAP high: 38.0 months (95\% Cl n.d.). (TIF 6349 kb)

Additional file 3: Figure S3. Kaplan-Meier curve showing OS of patients with either loss (A) or intact (B) arid1a expression (loss of expression describes scenarios of ARIDla-Gen alterations leading to a non-expressing protein - mainly related to mutation, deep deletion or promotormethylation of ARIDA1a-gene or loss (C) or intact (D) SWI/SNF stratified for XIAP low vs. high. (A) XIAP low: median OS 22.1 months (95\% CI 0 70.5 months) vs. XIAP high: 20.5 months ( $95 \%$ Cl 0-45.0 months). (B) XIAP low: median OS 30.5 months ( $95 \%$ CI 24.3-36.7 months) vs. XIAP high: 38.0 months (95\% Cl 24.7-51.3 months). (C) XIAP low: median OS 30.5 months (95\% Cl 16.8-44.2 months) vs. XIAP high: 20.5 months (95\% Cl 0-42.8 months). (D) XIAP low: median OS 32.6 months (95\% Cl 23.2-42.0 months) vs. XIAP high: 42.8 months (95\% Cl 12.3-73.3 months). (TIF 13550 kb)

Additional file 4: Figure S4. Representative images of XIAP stained tumor sections of either XIAP low or high expressing tumors. Scale bar indicates $50 \mu \mathrm{m}$. (TIF $4549 \mathrm{~kb}$ )

\section{Abbreviations}

EAC: Esophageal adenocarcinoma; ESCC: Esophageal squamous cell carcinoma; HR: Hazard ratio of death; TMA: Tissue-micro arrays; UICC: Union internationale contre le cancer; XIAP: X-linked inhibitor of apoptosis protein 


\section{Acknowledgements}

We acknowledge Arnulf H. Hölscher's work as the former chair of the Department of General, Visceral and Cancer Surgery, University of Cologne, who during that time operated a part of the studied patients.

\section{Funding}

LMS is supported by the Köln Fortune Programm, Faculty of Medicine, University of Cologne. HK acknowledges funding from the Deutsche Forschungsgemeinschaft (CRU 286, KA 2853/4-1 and CRC1218) and the Deutsche Krebshilfe (70112113). Though not specifically funding this study all funding bodies enabled the authors to design, analyse and interprete the data and write the manuscript.

\section{Availability of data and materials}

Datasets generated and analysed for this work are not publicly available but are accessible upon request.

\section{Authors' contributions}

Study design: $L M S, A Q$, FG; providing of study materials or samples: LMS, HK, $A Q, F G, C J B, T Z, H L, H F F, W S, M B$; data generation, analysis and interpretation: LMS, FG, PSP, AQ, HG, HL, JPW, FS; manuscript writing: all authors. All authors read and approved the final manuscript.

\section{Ethics approval and consent to participate}

The study was performed in agreement with the local research ethics guidelines (ethics committee medical faculty, University of Cologne). Human samples were collected at the University Hospital of Cologne with written informed consent concurring with the Declaration of Helsinki.

\section{Consent for publication}

Not applicable.

\section{Competing interests}

The authors declare that they have no competing interests.

\section{Publisher's Note}

Springer Nature remains neutral with regard to jurisdictional claims in published maps and institutional affiliations.

\section{Author details}

${ }^{1}$ Cologne Excellence Cluster on Cellular Stress Responses in Aging-Associated Diseases (CECAD), Center for Molecular Medicine Cologne (CMMC) and Institute for Medical Microbiology, Immunology and Hygiene, University of Cologne, CECAD Research Center, Joseph-Stelzmann-Str. 26, 50931 Cologne, Germany. ${ }^{2}$ Department of General, Visceral and Cancer Surgery, University of Cologne, Kerpener Str. 62, 50937 Cologne, Germany. ${ }^{3}$ Center for Integrated Oncology (CIO) Cologne Bonn, Kerpener Str. 62, 50924 Cologne, Germany. ${ }^{4}$ Institute of Pathology, University of Cologne, Kerpener Str. 62, 50937 Cologne, Germany. Department I of Internal Medicine, University of Cologne, Kerpener Str. 62, 50924 Cologne, Germany.

\section{Received: 19 February 2019 Accepted: 16 May 2019}

\section{Published online: 31 May 2019}

\section{References}

1. Lagergren J, Smyth E, Cunningham D, Lagergren P. Oesophageal cancer. Lancet. 2017;390(10110):2383-96.

2. Global Burden of Disease Cancer Collaboration, Fitzmaurice C, Dicker D, Pain A, Hamavid H, Moradi-Lakeh M, et al. The global burden of Cancer 2013. JAMA Oncol. 2015;1(4):505-27.

3. Kashkar H, Haefs C, Shin H, Hamilton-Dutoit SJ, Salvesen GS, Krönke $M$, et al. XIAP-mediated caspase inhibition in Hodgkin's lymphoma-derived B cells. J Exp Med. 2003;198(2):341-7.

4. Kashkar H. X-linked inhibitor of apoptosis: a Chemoresistance factor or a hollow promise. Clin Cancer Res. 2010;16(18):4496-502.

5. Obexer P, Ausserlechner MJ. X-linked inhibitor of apoptosis protein - a critical death resistance regulator and therapeutic target for personalized cancer therapy. Front Oncol. 2014;4(113):197.

6. Fulda S, Vucic D. Targeting IAP proteins for therapeutic intervention in cancer. Nat Rev Drug Discov. 2012;11(2):109-24.
7. Eckelman BP, Salvesen GS, Scott FL. Human inhibitor of apoptosis proteins: why XIAP is the black sheep of the family. EMBO Rep. 2006; 7(10):988-94.

8. Krieg A, Correa RG, Garrison JB, Le Negrate G, Welsh K, Huang Z, et al. XIAP mediates NOD signaling via interaction with RIP2. Proc Natl Acad Sci Natl Acad Sci. 2009;106(34):14524-9.

9. Andree M, Seeger JM, Schull S, Coutelle O, Wagner-Stippich D, Wiegmann $\mathrm{K}$, et al. BID-dependent release of mitochondrial SMAC dampens XIAP-mediated immunity against Shigella. EMBO J. 2014; 33(19):2171-87.

10. Lu M, Lin S-C, Huang Y, Kang YJ, Rich R, Lo Y-C, et al. XIAP induces NF-kB activation via the BIR1/TAB1 interaction and BIR1 dimerization. Mol Cell. 2007;26(5):689-702.

11. van Hagen P, Hulshof MCCM, van Lanschot JJB, Steyerberg EW, van Berge Henegouwen MI, Wijnhoven BPL, et al. Preoperative chemoradiotherapy for esophageal or junctional cancer. N Engl J Med. 2012;366(22):2074-84.

12. Al-Batran S-E, Homann N, Schmalenberg H, Kopp H-G, Haag GM, Luley KB, et al. Perioperative chemotherapy with docetaxel, oxaliplatin, and fluorouracil/leucovorin (FLOT) versus epirubicin, cisplatin, and fluorouracil or capecitabine (ECF/ECX) for resectable gastric or gastroesophageal junction (GEJ) adenocarcinoma (FLOT4AlO): a multicenter, randomized phase 3 trial. JCO. 2017;35(15_suppl): 4004-4.

13. Mönig SP, Schiffmann LM. [Resection of advanced esophagogastric adenocarcinoma : Extended indications]. Chirurg. 2016;87(5):398-405.

14. Cunningham D, Allum WH, Stenning SP, Thompson JN, Van de Velde $\mathrm{CJH}$, Nicolson $\mathrm{M}$, et al. Perioperative chemotherapy versus surgery alone for resectable gastroesophageal cancer. N Engl J Med. 2006; 355(1):11-20.

15. Simon R. Applications of tissue microarray technology. Methods Mol Biol. 2010;664:1-16.

16. Helbig D, Ihle MA, Pütz K, Tantcheva-Poor I, Mauch C, Büttner R, et al. Oncogene and therapeutic target analyses in atypical fibroxanthomas and pleomorphic dermal sarcomas. Oncotarget. 2016; 7(16):21763-74.

17. Plum PS, Gebauer F, Krämer M, Alakus H, Berlth F, Chon S-H, et al. HER2/neu (ERBB2) expression and gene amplification correlates with better survival in esophageal adenocarcinoma. BMC Cancer. 2019;19(1):38.

18. Quaas A, Heydt C, Gebauer F, Alakus H, Loeser H, Buettner R, et al. Genomic characterization of TP53-wild-type esophageal carcinoma. Transl Oncol. 2019:12(1):154-61.

19. Gao J, Aksoy BA, Dogrusoz U, Dresdner G, Gross B, Sumer SO, et al. Integrative analysis of complex Cancer genomics and clinical profiles using the cBioPortal. Sci Signal. 2013;6(269):pl1.

20. Cerami E, Gao J, Dogrusoz U, Gross BE, Sumer SO, Aksoy BA, et al. The cBio cancer genomics portal: an open platform for exploring multidimensional cancer genomics data. Cancer Discov. 2012;2(5): 401-4.

21. Schneider PM, Baldus SE, Metzger R, Kocher M, Bongartz R, Bollschweiler E, et al. Histomorphologic tumor regression and lymph node metastases determine prognosis following neoadjuvant Radiochemotherapy for esophageal Cancer. Ann Surg. 2005;242(5):684-92.

22. Jesinghaus M, Steiger K, Slotta-Huspenina J, Drecoll E, Pfarr N, Meyer P, et al. Increased intraepithelial CD3+ T-lymphocytes and high PD-L1 expression on tumor cells are associated with a favorable prognosis in esophageal squamous cell carcinoma and allow prognostic immunogenic subgrouping. Oncotarget. 2017;8(29):46756-68.

23. Dizdar $L$, Jünemann $L M$, Werner $T A$, Verde $P E$, Baldus $S E$, Stoecklein $N H$, et al. Clinicopathological and functional implications of the inhibitor of apoptosis proteins survivin and XIAP in esophageal cancer. Oncol Lett. 2018; 15(3):3779-89.

24. Zhang S, Ding F, Luo A, Chen A, Yu Z, Ren S, et al. XIAP is highly expressed in esophageal cancer and its downregulation by RNAi sensitizes esophageal carcinoma cell lines to chemotherapeutics. Cancer Biol Ther. 2014;6(6):974-9.

25. Silke J, Vaux DL. IAP gene deletion and conditional knockout models. Seminars in Cell and Developmental Biology. 2015;39:97-105.

26. Goncharov T, Hedayati S, Mulvihill MM, Izrael-Tomasevic A, Zobel K, Jeet S, et al. Disruption of XIAP-RIP2 association blocks NOD2- mediated inflammatory signaling. Mol Cell. 2018;69(4):551-7. 
27. Kashkar H, Seeger JM, Hombach A, Deggerich A, Yazdanpanah B,

Utermöhlen O, et al. XIAP targeting sensitizes Hodgkin lymphoma cells for cytolytic T-cell attack. Blood. 2006;108(10):3434-40.

28. Cancer Genome Atlas Research Network, Analysis working group: Asan University, BC Cancer Agency, Brigham and Women's hospital, Case Western Reserve University, Dana-Farber Cancer Institute, et al.

Integrated genomic characterization of oesophageal carcinoma. Nature. 2017:541(7636):169-75.

Ready to submit your research? Choose BMC and benefit from:

- fast, convenient online submission

- thorough peer review by experienced researchers in your field

- rapid publication on acceptance

- support for research data, including large and complex data types

- gold Open Access which fosters wider collaboration and increased citations

- maximum visibility for your research: over $100 \mathrm{M}$ website views per year

At $B M C$, research is always in progress.

Learn more biomedcentral.com/submissions 